\title{
A simple setup miniaturisation with multiple benefits for Green Chemistry in nanoparticle synthesis
}

\author{
Jette K. Mathiesen, ${ }^{a}{ }^{a}+$ Susan R. Cooper, ${ }^{a},+$ Andy S. Anker, ${ }^{a}$ Tiffany L. Kinnibrugh, ${ }^{b}$ \\ Kirsten M. $\varnothing$. Jensen ${ }^{\mathrm{a}, *}$ Jonathan Quinson ${ }^{\mathrm{a}, *}$
}

\begin{abstract}
a. Chemistry Department, University of Copenhagen, 5 Universitetsparken, 2100 Copenhagen, Denmark

b. X-ray Science Division, Advanced Photon Source, Argonne National Laboratory, Argonne, IL, USA

† These authors contributed equally.

*Corresponding authors: kirsten@chem.ku.dk, jonathan.quinson@chem.ku.dk
\end{abstract}

\begin{abstract}
The development of nanomaterials often relies on wet-chemical syntheses performed in reflux setups using round-bottom-flasks. An alternative approach to synthesise nanomaterials is presented that uses glass tubes designed for NMR analysis as reactors. It uses less solvent, generates less waste, provides safer conditions, is less prone to contamination and is compatible with high throughput screening.
\end{abstract}

\section{Introduction}

Nanomaterials (NMs) are used in multiple applications ranging from catalysis, optics, medicine or water/air treatments. ${ }^{1-3}$ Due to strong structure-property relations on the nanoscale, the careful and rational synthesis of a range of NMs is important. As a consequence, the development of controlled syntheses of NMs has been increasingly addressed as a key component of Green Chemistry. ${ }^{4-8}$

In particular, the need for protocols generating less waste and increasing safety during NM synthesis has been stressed.6,7 A focus is often given to the nature of solvents and reactants to use, or to alternative synthesis methods using for instance microwaves or ultrasound, that are considered more energy efficient. ${ }^{8}$ The development of new setups such as flow (micro)reactors has also proved useful to minimise waste and simplify the synthesis of NMs. ${ }^{9}, 10$ Such approaches are furthermore promising since they can be 
automatized and combined with emerging artificial intelligence-based decision-making processes, to rationally design new and/or improved NMs by resource and time efficient strategies. ${ }^{11}$ However, as stated by Volk et al., "despite these advantages, microfluidic systems have yet to be extensively adopted by the colloidal nanomaterial community". ${ }^{11}$ The above strategies often require specific equipment and expertise, which may account for their relatively limited implementation to date. Simpler alternative miniaturised systems with high throughput potential would gain to be proposed.

In wet chemical syntheses, preparing a wide range of NMs with various compositions, sizes and structures is possible, which results in tuned properties of the NMs to best match specific applications. ${ }^{6}$ The control of NM features is achieved by tuning experimental parameters, e.g. temperature, concentration of reactants, type of reactants or solvent composition. Wet-chemical methods often show promising scalability while being easily implemented in most modern laboratories ${ }^{12-14}$ for various applications. ${ }^{14-16}$ However, studying and understanding how synthetic parameters in a wet-chemical synthesis method influence the NM produced is often limited by the time and resources required to make a single batch of NMs.

Wet-chemical syntheses are typically inherited from organic or inorganic synthesis protocols and are often performed in round-bottom flasks in a reflux setup. In this textbook approach, $10-100 \mathrm{~mL}$ of solvent is typically required and only one experiment can be performed every few hours per setup, see Scheme 1. To understand how NM structures change with synthetic parameters, alternative high throughput strategies are needed.

We here propose a simple alternative to the conventional reflux setup, see Scheme 1. Instead of using relatively large volume flasks, we propose using glass tubes, designed for nuclear magnetic resonance (NMR) measurements. ${ }^{17,} 18$ The multiple advantages of this simple alternative in light of the Green Chemistry principles for NM synthesis ${ }^{6,8}$ are illustrated below with the synthesis of four different nanoparticles (NPs): gold ( $\mathrm{Au}$ ), iridium (Ir), osmium (Os) and copper sulfide $\left(\mathrm{Cu}_{2-\mathrm{x}} \mathrm{S}\right)$. Although precious metal based NPs are made of non-renewable resources, they remain key materials in multiple applications, ${ }^{7}$ e.g. to develop fossil-fuel free technologies for energy conversion, ${ }^{15}$ as well as models to understand NM formation. ${ }^{19}$ Even minor improvements in precious metal NP syntheses can have significant scientific, economic and ecologic impact. 


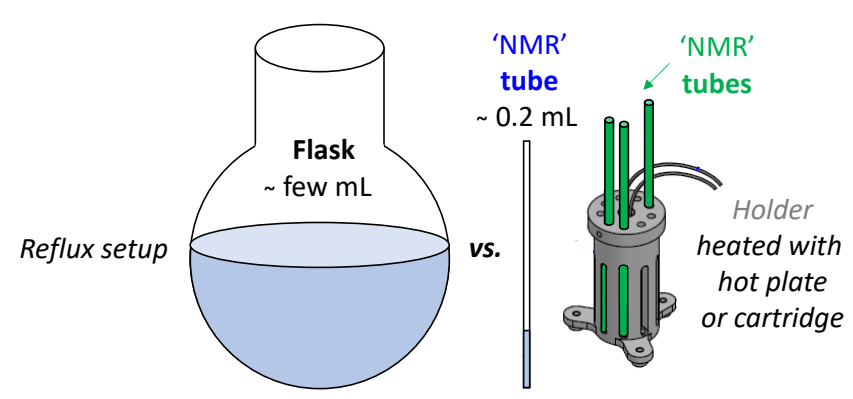

Scheme 1. Moving from round-bottom-flask to NMR tube glassware for greener NM syntheses and studies.

\section{Setup and benefits.}

Rather than using flasks to perform the synthesis of NMs, we use $3 \mathrm{~mm}$ diameter NMR tubes, see Materials and Methods in the Supplementary Information (SI), Figures S1-S2. This makes it possible to perform syntheses with lower volume: a round-bottom-flask will typically require a minimum of a few millilitres of solvent, whereas the NMR tubes can accommodate as low as $0.1 \mathrm{~mL}$, see Figure $\mathbf{S 3}$. This low volume reduces the amount of waste generated. It also allows to investigate the effect of high concentration solutions, as illustrated below with the example of Ir and Os NPs. So far, investigating synthetic conditions with high concentrations remains challenging. This is due to the cost of using large quantities of chemicals in the relatively large volumes preferred in reflux setups. By using high precursor concentrations, the volume needed to produce a given amount of material is reduced and the related waste generated is minismised. ${ }^{7,14}$

With the NMR tubes used as reactors, sample holders can be simply designed to perform several experiments at the same time (e.g. up to 9 experiments per holder in the current holder design) allowing high throughput screening, see Scheme 1 and Figures S1S2. The combination of a small volume of chemicals needed and the use of a dedicated holder allows safer operating conditions. As a result, both long(er) and an increased number of experiments can be performed with minimal lab space and little equipment required, while optimising the energy needed to heat up the solutions.

Temperature control is achieved by either using the temperature control of a standard heating plate or using dedicated heating cartridge with a temperature controller to control the heating rate, see Figures S1-S2 and the later example of $\mathrm{Cu}_{2-x} \mathrm{~S} N P s$ below. The use of septa to close the NMR tubes allows for controlling the atmosphere in the NMR tube, alternatively, nut and ferrules can be used for higher pressure experiments, see Figure S1. The length of the NMR tube provides an area of contact with the air to function under reflux conditions. This area can also be cooled down, e.g. with a fan or dedicated water-cooling devices, to allow reflux conditions. Among other practicalities, it is worth 
stressing that the synthesis can be performed with stirring by placing the cells on a stirring plate and simply using a commercially available stirrer bar (e.g. $8 \mathrm{~mm} \times 1.5 \mathrm{~mm}$ ) in the NMR tube, see Figure S3.

\section{Au NPs}

We first exemplify the use of the NMR tube setup for Au NPs. Au NMs are probably one of the most studied systems due to the multiple applications of $A u$ in catalysis, medicine, optics and many more. ${ }^{20-23}$ Au NPs have even been a case study for Green Chemistry in NM synthesis. ${ }^{7}$ In particular, the Turkevich synthesis is a widely used, studied and reported method where $\mathrm{HAuCl}_{4}$ at relatively low concentrations (ca. 0.1-0.5 mM) is reduced in water close to the boiling point by sodium tricitrate. ${ }^{24,}{ }^{25}$ Using the Turkevich method, several parameters influence the resulting size of the NPs such as temperature of synthesis, concentration of Au precursor, concentration of stabilizer and reducing agents trisodium citrate, injection of the gold precursor before or after heat treatment of the aqueous solution, among others. ${ }^{24}$ The synthesis is typically performed in ca. $200 \mathrm{~mL}$ of water. ${ }^{24}$

An optimisation of the above-mentioned parameters requires the design of an optimal Au NPs recipe. This task may be time consuming and is still mainly achieved by trial and error and/or systematic investigation of the effects of different parameters. In this respect, the use of miniaturised vessels can significantly save time and resources. To illustrate the relevance of scaling down the volume used, we compared the Au NPs obtained performing the Turkevich synthesis at $100{ }^{\circ} \mathrm{C}$ using $0.125 \mathrm{mM}^{\circ} \mathrm{HAuCl}_{4}$ and 2.2 $\mathrm{mM}$ trisodium citrate for 1 hour in $500 \mathrm{~mL}$ or $0.2 \mathrm{~mL}$. The transmission electron microscopy (TEM) micrographs of the materials obtained are displayed in Figure 1. The size of the NPs obtained is identical within the error of measurement: $13.1 \pm 3.9 \mathrm{~nm}$ in the NMR tube and $14.8 \pm 3.0 \mathrm{~nm}$ in the reflux setup. This is a first step and example of the suitability of NMR tubes for multi parametric studies. 


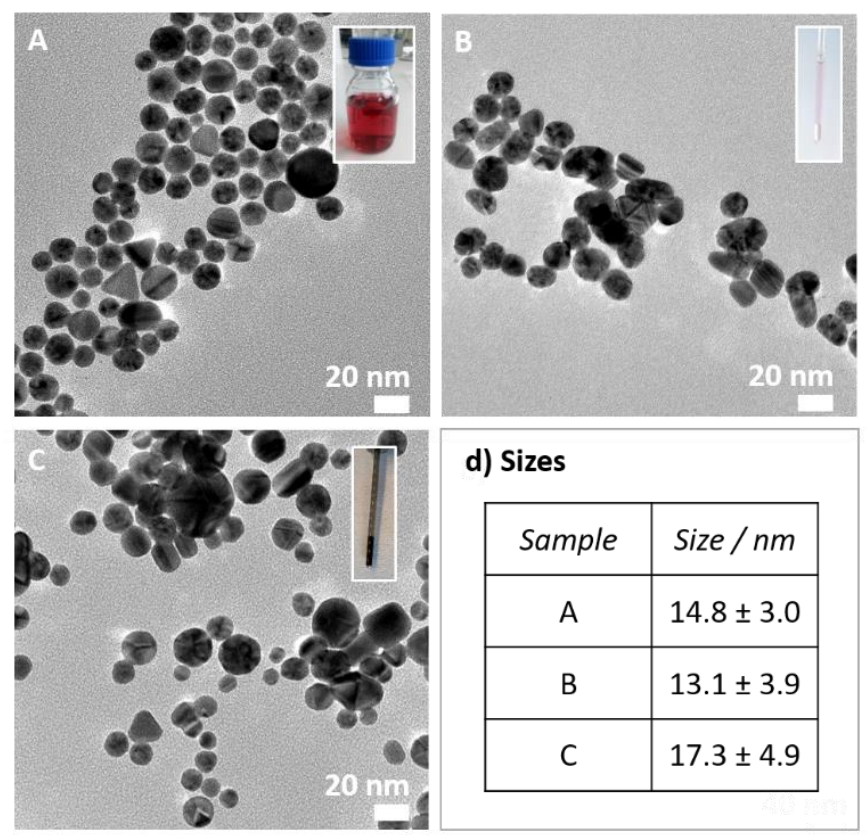

Figure 1. TEM micrographs of Au NPs obtained from $0.125 \mathrm{mM} \mathrm{HAuCl}_{4}$ in water in presence of $2.2 \mathrm{mM}$ trisodium citrate at $100{ }^{\circ} \mathrm{C}$ for 1 hour for a total volume of (A) $500 \mathrm{~mL}$ and (B) $0.2 \mathrm{~mL}$. (C) TEM micrographs of Au NPs obtained using oleylamine at $200{ }^{\circ} \mathrm{C}$ as solvent in $0.3 \mathrm{~mL}$. Insets are pictures of the resulting colloidal dispersions. TEM acquired at different magnifications and the related size distribution are available in Figure S4. (D) Table of the related size distributions.

A range of alternative Au NP syntheses use organic solvents such as oleylamine and relatively high temperature. ${ }^{26}$ Synthesesat higher temperature, e.g. $200{ }^{\circ} \mathrm{C}$, are also possible since the NMR tube can be closed with nut and ferrule. The synthesis leads to ca. $17 \mathrm{~nm}$ Au NPs, see Figure 1C.

\section{Ir NPs}

We reported a simple surfactant-free colloidal synthesis of precious metal NPs that is easily performed at low temperature $\left(<80^{\circ} \mathrm{C}\right)$ in alkaline mono-alcohols like methanol or ethanol. ${ }^{15}$ The absence of surfactant, the use of simple solvents and the low temperature already comply with the Green Chemistry principles for NP synthesis. 6, 7, 27 Moreover, the Ir NPs obtained by this method show high catalytic activity for the oxygen evolution reaction, ${ }^{15,} 28$ a key reaction to develop more sustainable energy conversion based on hydrogen as an energy carrier. The high catalytic activity is achieved without intensive washing or purification steps, thus complying further with the Green Chemistry principles for NP synthesis. ${ }^{6}$ 


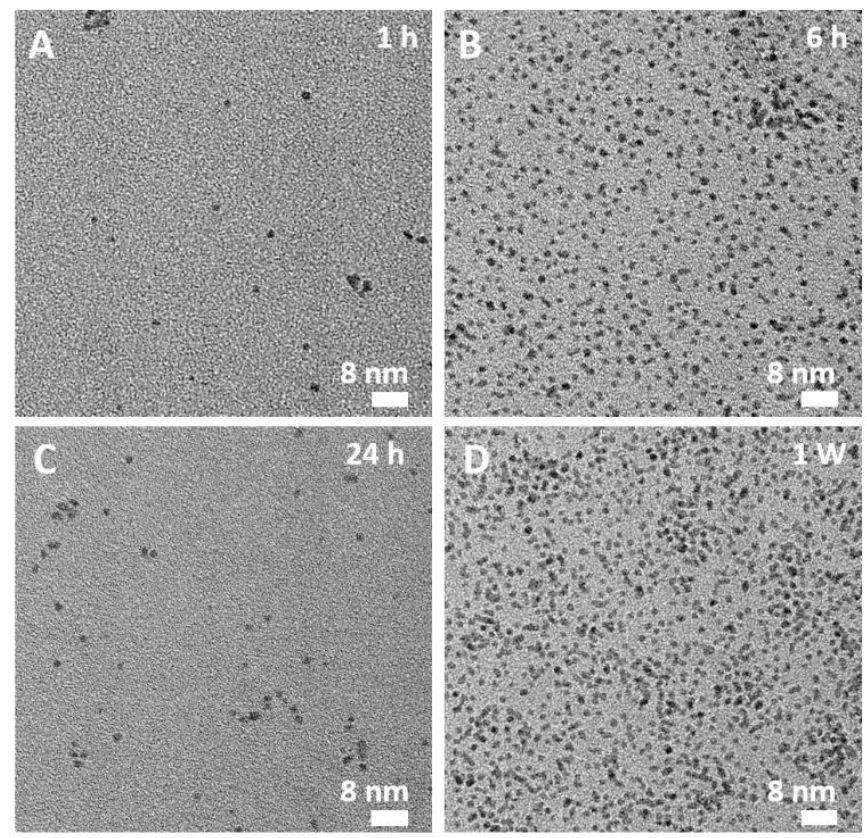

Figure 2. TEM micrographs or Ir NPs obtained from $100 \mathrm{mM} \mathrm{IrCl}_{3}$ in $1 \mathrm{M} \mathrm{NaOH}$ in methanol at $60{ }^{\circ} \mathrm{C}$ for a week for a total volume of $0.2 \mathrm{~mL}$. Aliquots were taken for analysis at different time of synthesis as indicated. Higher magnification micrographs and size distributions are available in Figures S5-S6.

When performing Ir NP synthesis, we observe by TEM analysis that the Ir NPs are extremely small in the range 1-2 nm across a wide range of experimental parameters. ${ }^{29} \mathrm{~A}$ legitimate question worth investigation is if raising the precursor concentration and/or increase the reaction time leads to a different NP size. Using a microwave synthesis or even a classical reflux setup, it is arguably challenging to perform high concentration and long-time reactions due to safety concerns, higher precursor cost and pressure on lab space and equipment access. Using NMR tubes as the reaction vessel, it is possible to perform safe and simple experiments using solutions with high precursor concentrations running for hours, days or weeks and therefore to easily perform high throughput and time resolved studies. Figure 2 gathers TEM micrographs of the NPs obtained in NMR tubes from a solution of $100 \mathrm{mM} \mathrm{IrCl}_{3}$ in $1 \mathrm{M} \mathrm{NaOH}$ in methanol after 1 hour, 6 hours, 24 hours or a week of synthesis, see also Figure S3. NPs with an average size of $1.6 \mathrm{~nm}$ are obtained, see also Figures S5-S6. These results show that small Ir NPs are obtained even at high precursor concentration and relatively long reaction time, and so illustrate the robustness of the synthesis to produce small and surfactant-free Ir NPs with a high surface available for catalytic application, using a relatively green synthesis approach, ${ }^{27}$ compatible with industrial requirements. ${ }^{15,} 28$ The synthesis method shows promising 
features for scalability, whether by increasing the precursor concentration and/or reducing the volume of solution, see SI for more discussion.

\section{Os NPs}

Due to their scarcity, some precious metals have received less attention than others. There are relatively few reports on Os NP synthesis. ${ }^{30-32}$ Taking advantage of the small volume of solvent needed, we investigated the surfactant-free synthesis of Os NPs using a high precursor concentration in simple low boiling points solvents such as a mixture of methanol and water. Figure $\mathbf{3}$ is a TEM micrograph of the resulting materials obtained for a one-week long synthesis starting with $100 \mathrm{mM}$ of $\mathrm{OsCl}_{3}$. Small size NPs, ca. $1.6 \pm 0.4 \mathrm{~nm}$ in diameter, are obtained. Although Os NPs are made of non-earth abundant element, the small size Os NPs obtained by a surfactant-free approach are relevant to develop more sustainable energy conversion systems to move away from fossil-fuels based energy. ${ }^{33-35}$

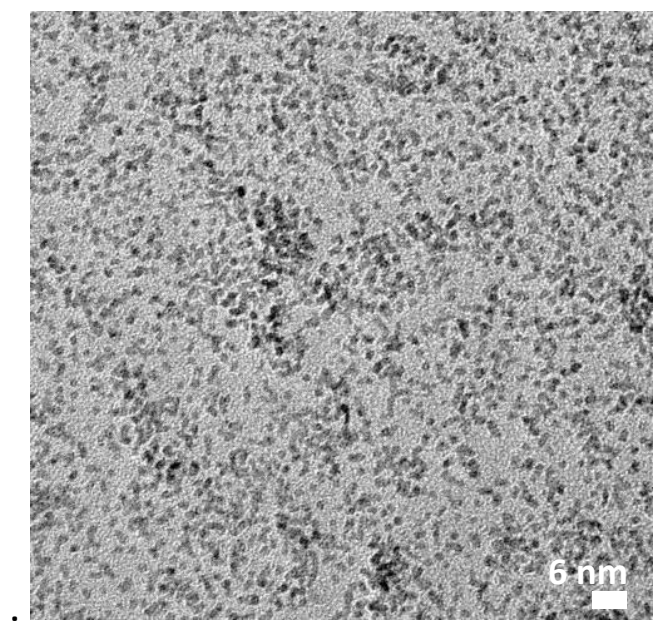

Figure 3. TEM micrographs of Os NPs obtained from $100 \mathrm{mM} \mathrm{OsCl}_{3}$ in methanol:water $(1: 2, v: v)$ at $85^{\circ} \mathrm{C}$ for a week, for a total volume of $0.2 \mathrm{~mL}$. Size distribution are available in Figure $\mathbf{S 7}$.

\section{$\mathrm{Cu}_{2-\mathrm{x}} \mathrm{S} \mathrm{NPS}$}

To illustrate the broad applicability of NMR tubes as reaction vessels, the synthesis of $\mathrm{Cu}_{2-x} \mathrm{~S}$ NPs was also performed as detailed in $\mathrm{SI} \mathrm{Cu}_{2-\mathrm{x}} \mathrm{S}$ NPs have applications in batteries, ${ }^{36}$, ${ }^{37}$ sensors $^{38}$ and as an oxygen evolution reaction catalyst. ${ }^{39}$ The analysis with a lab-source XRD instrument of the NPs synthesized in the NMR tubes suggests that small $\mathrm{Cu}_{2-\mathrm{XS}} \mathrm{NPs}$ were formed, see Figure 4A. TEM analysis shows a size of $4.5 \pm 0.9 \mathrm{~nm}$, see Figure 4B and 
Figure S8. While some features are observed in the XRD pattern in Figure 4A, the broadening of Bragg peaks from these small NPs makes any structural investigation challenging. In contrast, the use of the NMR tube makes further characterization straightforward, e.g. using synchrotron scattering techniques, as many measurements can be done directly on the sample in the NMR tube. Here, the as-prepared samples in closed NMR tubes were sent to beamline 11-ID-B at Argonne National Laboratory for characterisation by X-ray total scattering with pair distribution function (PDF) analysis. The PDF can be understood as a histogram of atom-atom distances in the material. In Figure 4C and $D$ are reported the $F(Q)$ (data in reciprocal space) and $G(r)$ (data in real space) of the reaction solution after background subtraction. The PDF analysis confirm that the NPs can be described by a $\beta$-chalcocite model in space group $\mathrm{P}_{3} / \mathrm{mmc}^{40}$ with deviations in the local structure, see Figure $\mathbf{5 9 .}$
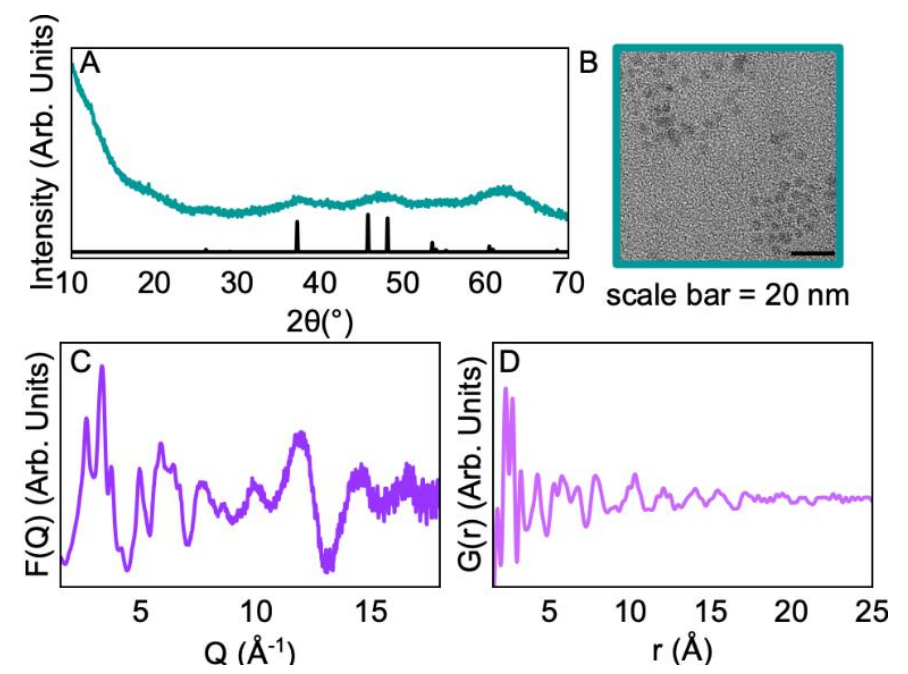

Figure 4. Characterisation of $\mathrm{Cu}_{2-x} \mathrm{~S}$ NPs obtained in an NMR tube setup. (A) Using a labsource XRD instrument (blue) compared to a calculated diffraction pattern of $\beta$-chalcocite taking the space group $\mathrm{P}_{3} / \mathrm{mmc}$ (black). (B) TEM micrographs of the washed NPs. Scale bar is $20 \mathrm{~nm}$. (C) Displays of the $F(Q)$ and (D) PDF $(G(r))$ of X-ray total scattering data collected at Argonne national lab at beamline 11-ID-B.

\section{Further technical and practical considerations.}

As illustrated above, measurements performed at synchrotrons offer unique opportunities to advance material sciences. ${ }^{41}$ However, a drawback is that syntheses developed and optimized in laboratories are often challenging to adapt to the requirements and limitations of synchrotron setups. For example, PDF measurements are best performed on samples with high concentration of the material of interest, especially 
when considering poorly scattering elements like transition metal sulfides. Here, the NMR tube setup offers the option to readily investigate high concentration of materials during parametric studies performed in home laboratories (outside synchrotrons facilities). Ultimately, this allows comparing more directly selected measurements performed at synchrotron facilities with larger screening experiments done in a chemistry lab. This further enables researchers to design experiments for even more complex in situ analysis of various reactions. ${ }^{17,42}$

NMR tubes make it possible to use a clean vessel playing the role of reactor for each experiment. This alleviates the need to clean glassware between each experiment, thus limiting the chemical waste generation related to cleaning steps, e.g. using aqua regia in the case of Au NPs synthesis. ${ }^{21}$ More importantly, it alleviates the question of cross contamination, thus addressing the well-known issue of reproducibility in NM science, often ascribed to chemical impurities, ${ }^{43}$ which can also result from different cleaning procedures. Further variables to account for a lack of reproducibility in NM synthesis can be variations of room temperature or room light, as well as the general stability overtime of the chemicals and/or precursors solutions. Indeed, these variables may be challenging to control across long periods of time when a study would last for instance several weeks of months. ${ }^{44}$ This drawback is alleviated in the present approach since several experiments including controls can be performed at the same time. Energy is saved because several experiments are heated all at once. The ease of simply performing relatively long experiments is also a positive feature towards improved yield of the precursor conversion to NMs.

It could be argued that due to the small diameter of the NMR tubes, capillary effects might come into play and the actual temperature-pressure during reaction might not be well-known. This issue of variability in physical parameters of reactors, e.g. heat transfer properties, stirring etc., is the same for most scaling up to date, e.g. when moving from reflux-setups to larger scale reactors. In this respect, the use of NMR tubes reactors remains a convenient and green approach to screen the influence of experimental parameters.

A final practical consideration is storage and reuse. The small NMR tubes are easily stored due to their small diameter and length. The $18 \mathrm{~cm}$ long NMR tube can easily be cut with a commercially available glasscutter to save even more space. The open end of the glass can be sealed by melting with a torch (e.g. butane torch), for instance when toxic reagents and/or air sensitive compounds are involved. The samples can be safely and space-efficiently stored for further analysis, e.g. for shipping to synchrotrons. Additionally, the cut section of the NMR tube can be cleaned and sealed by melting one extremity to be re-used as a new miniaturised vessel. 


\section{Conclusions}

A simple alternative to the classical round-bottom flask synthesis approach to prepare NMs is presented. By using commercially available NMR tubes, a miniaturised vessel suitable for the synthesis of various NMs is readily obtained. This approach complies with several of the principles of Green Chemistry for NM synthesis. ${ }^{6,7}$ The miniaturised vessel offers safer conditions, lower volume, reduced waste generation and minimise the use of lab space to perform NM synthesis. Several experiments can be performed in parallel, thus also saving energy and increasing reproducibility. This approach is therefore directly relevant for academic research and research and development but also educational purposes.

With the increasing interest in machine learning and artificial intelligence, large datasets are needed to feed algorithms. ${ }^{45}$ The present approach allows for high throughput screening. Characterisation remains a limiting factor - although we show that enough material is obtained for characterisation like TEM, XRD or PDF and naturally NMR characterisation - but the variety of approaches and the expected improvements in this area of research are promising. ${ }^{6,17,46}$ It is expected that the simple alternative proposed here will also be relevant for various syntheses of molecules and other chemical reactions.

\section{Acknowledgement}

This project has received funding from the European Union's Horizon 2020 research and innovation programme under the Marie Sklodowska-Curie grant agreement No. 840523 (CoSolCat, JQ) and 841903 (SRC). We are grateful to the Villum Foundation for financial support through a Villum Young Investigator grant (VKR00015416). This work is part of a project that has received funding from the European Research Council (ERC) under the European Union's Horizon 2020 Research and Innovation Programme (grant agreement No. 804066). We acknowledge the Danish National Research Foundation (DNRF 149) Center for High-Entropy Alloy Catalysis (CHEAC). This research used resources of the Advanced Photon Source, a U.S. Department of Energy (DOE) Office of Science User Facility, operated for the DOE Office of Science by Argonne National Laboratory under Contract No. DE-AC02-06CH11357 (GUP-73929). E.T.S. Kjær, B. Wang, O. Aalling-Frederiksen, R. Pittkowski, University of Copenhagen, Denmark, H-C. Lu, University of Texas at Austin, USA, and beamline scientists O.J. Borkiewicz and L.C. Gallington are thanked for their help on synchrotron beamtime. S.B. Simonsen and L. Theil Kuhn, Technical University of Denmark, Denmark, are thanked for access to TEM facilities. Morten Liborius Jensen from the University of Copenhagen workshop is thanked for his help on the cell holder design and manufacturing. 


\section{References}

1. Cargnello, M., Colloidal Nanocrystals as Building Blocks for Well-Defined Heterogeneous Catalysts. Chemistry of Materials 2019, 31 (3), 576-596.

2. Kalus, M. R.; Rehbock, C.; Barsch, N.; Barcikowski, S., Colloids created by light: Lasergenerated nanoparticles for applications in biology and medicine. Materials Today-Proceedings 2017, 4, S93-S100.

3. Chaudhary, S.; Sharma, P.; Chauhan, P.; Kumar, R.; Umar, A., Functionalized nanomaterials: a new avenue for mitigating environmental problems. International Journal of Environmental Science and Technology 2019, 16 (9), 5331-5358.

4. Schmidt, J.; Marques, M. R. G.; Botti, S.; Marques, M. A. L., Recent advances and applications of machine learning in solid-state materials science. Npj Computational Materials 2019, 5, 83.

5. Dhand, C.; Dwivedi, N.; Loh, X. J.; Ying, A. N. J.; Verma, N. K.; Beuerman, R. W.; Lakshminarayanan, R.; Ramakrishna, S., Methods and strategies for the synthesis of diverse nanoparticles and their applications: a comprehensive overview. RSC Advances 2015, 5 (127), 105003-105037.

6. Hutchison, J. E., The Road to Sustainable Nanotechnology: Challenges, Progress and Opportunities. ACS Sustainable Chemistry \& Engineering 2016, 4 (11), 5907-5914.

7. Gilbertson, L. M.; Zimmerman, J. B.; Plata, D. L.; Hutchison, J. E.; Anastas, P. T., Designing nanomaterials to maximize performance and minimize undesirable implications guided by the Principles of Green Chemistry. Chemical Society Reviews 2015, 44 (16), 5758-5777.

8. Duan, H. H.; Wang, D. S.; Li, Y. D., Green chemistry for nanoparticle synthesis. Chemical Society Reviews 2015, 44 (16), 5778-5792.

9. Sui, J. S.; Yan, J. Y.; Liu, D.; Wang, K.; Luo, G. S., Continuous Synthesis of Nanocrystals via Flow Chemistry Technology. Small 2020, 16 (15), 1902828.

10. Suryawanshi, P. L.; Gumfekar, S. P.; Bhanvase, B. A.; Sonawane, S. H.; Pimplapure, M. S., A review on microreactors: Reactor fabrication, design, and cutting-edge applications. Chemical Engineering Science 2018, 189, 431-448.

11. Volk, A. A.; Epps, R. W.; Abolhasani, M., Accelerated Development of Colloidal Nanomaterials Enabled by Modular Microfluidic Reactors: Toward Autonomous Robotic Experimentation. Advanced Materials, 33, 2004495.

12. Saldanha, P. L.; Lesnyak, V.; Manna, L., Large scale syntheses of colloidal nanomaterials. Nano Today 2017, 12, 46-63.

13. van Embden, J.; Chesman, A. S. R.; Jasieniak, J. J., The Heat-Up Synthesis of Colloidal Nanocrystals. Chemistry of Materials 2015, 27 (7), 2246-2285.

14. Quinson, J.; Jensen, K. M. O., From platinum atoms in molecules to colloidal nanoparticles: A review on reduction, nucleation and growth mechanisms. Advances in Colloid and Interface Science 2020, 286, 102300. 
15. Quinson, J.; Neumann, S.; Wannmacher, T.; Kacenauskaite, L.; Inaba, M.; Bucher, J.; Bizzotto, F.; Simonsen, S. B.; Kuhn, L. T.; Bujak, D.; Zana, A.; Arenz, M.; Kunz, S., Colloids for Catalysts: A Concept for the Preparation of Superior Catalysts of Industrial Relevance. Angewandte Chemie International Editon 2018, 57 (38), 12338-12341.

16. Losch, P.; Huang, W. X.; Goodman, E. D.; Wrasman, C. J.; Holm, A.; Riscoe, A. R.; Schwalbe, J. A.; Cargnello, M., Colloidal nanocrystals for heterogeneous catalysis. Nano Today 2019, 24, 15-47.

17. Marbella, L. E.; Millstone, J. E., NMR Techniques for Noble Metal Nanoparticles. Chemistry of Materials 2015, 27 (8), 2721-2739.

18. Mateo, J. M.; de la Hoz, A.; Uson, L.; Arruebo, M.; Sebastian, V.; Gomez, M. V., Insights into the mechanism of the formation of noble metal nanoparticles byin situNMR spectroscopy. Nanoscale Advances 2020, 2 (9), 3954-3962.

19. Handwerk, D. R.; Shipman, P. D.; Whitehead, C. B.; Ozkar, S.; Finke, R. G., MechanismEnabled Population Balance Modeling of Particle Formation en Route to Particle Average Size and Size Distribution Understanding and Control. Journal of the American Chemical Society 2019, 141 (40), 15827-15839.

20. Villa, A.; Dimitratos, N.; Chan-Thaw, C. E.; Hammond, C.; Veith, G. M.; Wang, D.; Manzoli, M.; Prati, L.; Hutchings, G. J., Characterisation of gold catalysts. Chemical Society Reviews 2016, 45 (18), 4953-4994.

21. De Souza, C. D.; Nogueira, B. R.; Rostelato, M., Review of the methodologies used in the synthesis gold nanoparticles by chemical reduction. Journal of Alloys and Compounds 2019, 798, 714-740.

22. Gupta, A.; Pandey, S.; Yadav, J. S., A Review on Recent Trends in Green Synthesis of Gold Nanoparticles for Tuberculosis. Advanced Pharmaceutical Bulletin 2021, 11 (1), 10-27.

23. Chakraborty, I.; Pradeep, T., Atomically Precise Clusters of Noble Metals: Emerging Link between Atoms and Nanoparticles. Chemical Reviews 2017, 117 (12), 8208-8271.

24. Wuithschick, M.; Birnbaum, A.; Witte, S.; Sztucki, M.; Vainio, U.; Pinna, N.; Rademann, K.; Emmerling, F.; Kraehnert, R.; Polte, J., Turkevich in New Robes: Key Questions Answered for the Most Common Gold Nanoparticle Synthesis. ACS Nano 2015, 9 (7), 7052-7071.

25. Turkevich, J.; Stevenson, P. C.; Hillier, J., A Study of the Nucleation and Growth Processes in the Synthesis of Colloidal Gold.. Discussions of the Faraday Society 1951, (11), 55-56.

26. Hiramatsu, H.; Osterloh, F. E., A simple large-scale synthesis of nearly monodisperse gold and silver nanoparticles with adjustable sizes and with exchangeable surfactants. Chemistry of Materials 2004, 16 (13), 2509-2511.

27. Quinson, J.; Kacenauskaite, L.; Bucher, J.; Simonsen, S. B.; Kuhn, L. T.; Oezaslan, M.; Kunz, S.; Arenz, M., Controlled Synthesis of Surfactant-Free Water-Dispersible Colloidal Platinum Nanoparticles by the Co4Cat Process. ChemSusChem 2019, 12 (6), 1229-1239. 
28. Bizzotto, F.; Quinson, J.; Zana, A.; Kirkensgaard, J.; Dworzak, A.; Oezaslan, M.; Arenz, M., Ir nanoparticles with ultrahigh dispersion as oxygen evolution reaction (OER) catalyst: synthesis and activity benchmarking. Catalysis Science \& Technology 2019, 9, 6345-6356.

29. Quinson, J.; Kacenauskaite, L.; Schroder, J.; Simonsen, S. B.; Kuhn, L. T.; Vosch, T.; Arenz, M., UV-induced syntheses of surfactant-free precious metal nanoparticles in alkaline methanol and ethanol. Nanoscale Advances 2020, 2 (6), 2288-2292.

30. Wakisaka, T.; Kusada, K.; Yamamoto, T.; Toriyama, T.; Matsumura, S.; Ibrahima, G.; Seo, O.; Kim, J.; Hiroi, S.; Sakata, O.; Kawaguchi, S.; Kubota, Y.; Kitagawa, H., Discovery of facecentred cubic Os nanoparticles. Chemical Communications 2020, 56 (3), 372-374.

31. Hirai, H.; Nakao, Y.; Toshima, N., PREPARATION OF COLLOIDAL TRANSITION-METALS IN POLYMERS BY REDUCTION WITH ALCOHOLS OR ETHERS. Journal of Macromolecular ScienceChemistry 1979, A13 (6), 727-750.

32. Pitto-Barry, A.; Perdigao, L. M. A.; Walker, M.; Lawrence, J.; Costantini, G.; Sadler, P. J.; Barry, N. P. E., Synthesis and controlled growth of osmium nanoparticles by electron irradiation. Dalton Transactions 2015, 44 (47), 20308-20311.

33. Lim, C. S.; Sofer, Z.; Toh, R. J.; Eng, A. Y. S.; Luxa, J.; Pumera, M., Iridium- and Osmiumdecorated Reduced Graphenes as Promising Catalysts for Hydrogen Evolution. ChemPhysChem 2015, 16 (9), 1898-1905.

34. Lam, V. W. S.; Gyenge, E. L., High-performance osmium nanoparticle electrocatalyst for direct borohydride PEM fuel cell anodes. Journal of the Electrochemical Society 2008, 155 (11), B1155-B1160.

35. Danilovic, N.; Subbaraman, R.; Chang, K.-C.; Chang, S. H.; Kang, Y. J.; Snyder, J.; Paulikas, A. P.; Strmcnik, D.; Kim, Y.-T.; Myers, D.; Stamenkovic, V. R.; Markovic, N. M., Activity-Stability Trends for the Oxygen Evolution Reaction on Monometallic Oxides in Acidic Environments. Journal of Physical Chemistry Letters 2014, 5 (14), 2474-2478.

36. Jiang, K.; Chen, Z. H.; Meng, X. B., CuS and Cu2S as Cathode Materials for Lithium Batteries: A Review. ChemElectroChem 2019, 6 (11), 2825-2840.

37. Rui, X. H.; Tan, H. T.; Yan, Q. Y., Nanostructured metal sulfides for energy storage. Nanoscale 2014, 6 (17), 9889-9924.

38. Guo, M. R.; Law, W. C.; Liu, X.; Cai, H. X.; Liu, L. W.; Swihart, M.; Zhang, X. H.; Prasad, P. N., Plasmonic Semiconductor Nanocrystals as Chemical Sensors: $\mathrm{Pb}^{2+}$ Quantitation via Aggregation-Induced Plasmon Resonance Shift. Plasmonics 2014, 9 (4), 893-898.

39. Wang, X. L.; Ke, Y. J.; Pan, H. Y.; Ma, K.; Xiao, Q. Q.; Yin, D. Q.; Wu, G.; Swihart, M. T., Cu-Deficient Plasmonic Cu2-xS Nanoplate Electrocatalysts for Oxygen Reduction. ACS Catalysis 2015, 5 (4), 2534-2540.

40. Cava, R. J.; Reidinger, F.; Wuensch, B. J., Mobile ion Distribution and Anharmonic Thermal otion in Fast lon Conducting $\mathrm{Cu}_{2} \mathrm{~S}$. Solid State Ionics 1981, 5 (OCT), 501-504. 
41. Wu, S. Y.; Li, M. R.; Sun, Y. G., In Situ Synchrotron X-ray Characterization Shining Light on the Nucleation and Growth Kinetics of Colloidal Nanoparticles. Angewandte ChemieInternational Edition 2019, 58 (27), 8987-8995.

42. Mathiesen, J. K.; Quinson, J.; Dworzak, A.; Vosch, T.; Juelsholt, M.; Kjær, E. T. S.; Schr ö der, J.; Kirkensgaard, J. J. K.; Oezaslan, M.; Arenz, M.; Jensen , K. M. Ø., Insights from In Situ Studies on the Early Stages of Platinum Nanoparticle Formation. Journal of Physical Chemistry Letters 2021, 12 (12), 3224-3231.

43. Liz-Marzan, L. M.; Kagan, C. R.; Millstone, J. E., Reproducibility in Nanocrystal Synthesis? Watch Out for Impurities! ACS Nano 2020, 14 (6), 6359-6361.

44. Quinson, J.; Mathiesen, J. K.; Schroder, J.; Dworzak, A.; Bizzotto, F.; Zana, A.; Simonsen, S. B.; Kuhn, L. T.; Oezaslan, M.; Jensen, K. M. O.; Arenz, M., Teaching old precursors new tricks: Fast room temperature synthesis of surfactant-free colloidal platinum nanoparticles. Journal of Colloid and Interface Science 2020, 577, 319-328.

45. Kalinin, S. V.; Sumpter, B. G.; Archibald, R. K., Big-deep-smart data in imaging for guiding materials design. Nature Materials 2015, 14 (10), 973-980.

46. Modena, M. M.; Ruhle, B.; Burg, T. P.; Wuttke, S., Nanoparticle Characterization: What to Measure? Advanced Materials 2019, 31 (32), 1901556. 